Doreen Fiedler, Franziska Bonatz, Lorena Koch und Natascha Michel

\title{
Deutsche Stiftung für junge Erwachsene mit Krebs
}

In:

Philip van der Eijk / Detlev Ganten / Roman Marek (Hrsg.): Was ist Gesundheit? :

Interdisziplinäre Perspektiven aus Medizin, Geschichte und Kultur

ISBN: 978-3-11-071333-6. - Berlin / Boston: De Gruyter, 2021

(Humanprojekt : Interdisziplinäre Anthropologie ; 18)

Teil 5: Gesundheit aus Sicht der Erkrankten und Angehörigen

S. $490-493$ 


\section{Doreen Fiedler, Franziska Bonatz, Lorena Koch und Natascha Michel}

\section{Deutsche Stiftung für junge Erwachsene mit Krebs}

Die Diagnose Krebs ist für jeden Betroffenen ein einschneidendes Erlebnis, aber in einem so jungen Lebensalter ist es besonders dramatisch. Viele Gedanken sind schon bei Diagnosestellung von enormer Bedeutung: Kann ich meine Ausbildung jemals beenden? Wann kann ich wieder in die Welt und etwas erleben? Kann ich jemals noch Kinder bekommen? Auch finanzielle Sorgen und die Angst des sozialen Abstiegs rücken in den Vordergrund. Dass man in seiner Altersklasse nicht alleine ist, zeigen Studien. Jedes Jahr erkranken etwa 16.500 junge Menschen zwischen 18 und 39 Jahre an Krebs.

Die Therapie beginnt und man findet sich plötzlich neben vielen älteren Mitpatienten wieder. Man versucht, sich alle Anweisungen der Krankenschwestern zu merken: Diese Tablette morgens, diese abends, und man bekommt den Ratschlag: „Wenn es Ihnen schlecht wird, dann haben wir hier noch ein Medikament für Sie“. Unweigerlich lauscht man aber auch den Gesprächen der meistens Ü60-Mitbetroffenen, die sich vor allem um folgende Themen drehen: Rente, Enkelkinder, Kochrezepte und der Rollator. Ohne Zweifel essenzielle Thematiken im betagten Alter, welche aber nicht die Lebenswelt eines jungen Betroffenen widerspiegeln.

Aus dieser Not heraus recherchierte ich im Internet und wurde schnell auf die Deutsche Stiftung für junge Erwachsene mit Krebs aufmerksam. Die Stiftung möchte durch die Förderung von Wissenschaft und Forschung sowie des öffentlichen Gesundheitswesens junge Menschen mit Krebs während und auch nach ihrer Erkrankung unterstützen und begleiten. Sie versteht sich als Ansprechpartnerin für alle Fragen von PatientInnen, Angehörigen, WissenschaftlerInnen, UnterstützerInnen und der Öffentlichkeit. Die Deutsche Stiftung für junge Erwachsene mit Krebs wurde 2014 durch die DGHO Deutsche Gesellschaft für Hämatologie und Medizinische Onkologie e.V. gegründet und setzt sich seitdem für die Verbesserung der Versorgungssituation junger Erwachsener mit Krebs ein.

Genau die richtige Anlaufstelle für meine Fragestellungen. Ich traf mich kurz nach der Diagnose mit einigen Betroffenen beim TREFFPUNKT Heidelberg - einem sogenannten Stammtisch für junge Erwachsene mit Krebs. Neben ganz praktischen Fragestellungen, wie z.B. beantrage ich einen Schwerbehindertenausweis und was haben die Merkzeichen für eine Relevanz, waren auch die persönlichen Gespräche interessant, aufschlussreich und durch keine wissen-

Ә OpenAccess. (C 2021 Doreen Fiedler, Franziska Bonatz, Lorena Koch und Natascha Michel, publiziert von De Gruyter. (cc) BY-NC-SA Dieses Werk ist lizenziert unter einer Creative Commons Namensnennung - Nicht kommerziell - Weitergabe unter gleichen Bedingungen 4.0 International Lizenz. 
schaftliche Publikation zu ersetzen. Die gemeinsamen Treffen, Projekte und Angebote sieht die Stiftung als ganzheitliche Unterstützung von Betroffenen, Angehörigen und MultiplikatorInnen und sie stellen eine umfassende Ergänzung zu bestehenden Angeboten des Versorgungssystems dar. Dabei ist besonders wichtig hervorzuheben, dass sie niederschwellig und kostenfrei sind und auf die Aktivierung der Betroffenen und ihrer Umgebung zielen. Neben der Hilfe zur Selbsthilfe werden auch anwaltschaftliche Funktionen ausgeübt, um die Patientenrechte der jungen Betroffenen zu stärken.

Durch einige Projekte stieg das Bedürfnis, sich ehrenamtlich zu engagieren und die Öffentlichkeit über die Themen der Erkrankung meiner Altersgruppe aufzuklären. Aber auch der Wunsch, Versorgungslücken nicht nur aufzudecken, sondern auch darauf hinzuweisen, war von essenzieller Bedeutung. Dank umfangreicher Unterstützung wurde nicht nur eine Anlaufstelle, sondern auch eine mehrstufige Plattform für Informations- und Hilfsangebote geschaffen. Weiterhin wurde der Patient:innenbeirat gegründet, der die Belange aus einer anderen Sichtweise betrachtet und das Kuratorium der Stiftung in wichtigen Angelegenheiten berät.

Auch in der aktuellen Corona-Pandemie steht die Stiftung jungen Betroffenen zur Seite, beantwortet Fragen und bietet online Webinare zu dem Thema an. Denn was viele vergessen ist, dass wir jungen Patienten momentan nicht nur wegen unseren eigentlichen Krankheiten einiges zu bewältigen haben, wir müssen uns zusätzlich auch vor dem Virus schützen, da auch wir trotz unseres jungen Alters zur Risikogruppe gehören.

Eines der wichtigsten Projekte, dass die Stiftung seit 2017 gemeinsam mit ihrer Stifterin betreut, ist der Einsatz für die Kostenübernahme der fruchtbarkeitserhaltenden Maßnahmen bei jungen Betroffenen durch die gesetzlichen Krankenkassen. Die durchzuführenden Therapien führen oftmals zur Unfruchtbarkeit. Viele Betroffene müssen sich noch am Diagnosetag über das weitere Vorgehen entscheiden und sind schlichtweg damit überfordert. Zudem sind die Kosten für fruchtbarkeitserhaltende Maßnahmen sehr hoch, was gerade in unserem jungen Alter dazu führen kann, dass man es sich entweder schlichtweg nicht leisten kann oder sogar Kredite dafür aufgenommen werden müssen.

Insbesondere finanzielle Sorgen stehen in der Spitzengruppe der Beeinträchtigungen der Lebensqualität junger Betroffener. Im Mai 2019 trat auf das Wirken der Stiftung hin ein entsprechendes Gesetz in Kraft. Die dazugehörige Richtlinie des Gemeinsamen Bundesausschusses (G-BA) wurde im Juli 2020 beschlossen. Seit dem Gesetzesbeschluss im Mai 2019 berät die Stiftung junge Betroffene bei Fragen rund um die Kostenübernahme und unterstützt bei der Formulierung von Anträgen und Widersprüchen. Auch hier wird die Öffentlichkeit mit aktiver Pressearbeit für das Thema sensibilisiert und es wird regelmäßig an 
beteiligte Akteure herangetreten. Durch die aktive Mitarbeit von uns PatientInnen werden Problemfelder gezielt identifiziert.

In einer im September 2019 gemeinsam mit der DGHO erstellten Schriftenreihe wurden aktuelle Hintergründe dazu untersucht. Der Band gibt einen Überblick über finanzielle Belastungen, Lücken in der sozialen Absicherung und macht konkrete Vorschläge für Verbesserungen. Zusätzlich wurde in diesem Jahr eine Arbeitsgruppe mit einigen Betroffenen ins Leben gerufen, die hilfreiche Tipps und Tricks im Umgang mit der Ausbildung, dem Studium oder dem Job sammelt, aufbereitet und online auf der Webseite der Stiftung veröffentlicht. Somit sind jederzeit alle relevanten Informationen schnell und übersichtlich für jedermann verfügbar.

Darüber hinaus fordert die Stiftung gegenüber der Politik die Erschließung der verstreuten Datenbestände, die Förderung der Forschung auf diesem Gebiet und konkrete Schritte zur Verbesserung der finanziellen und sozialen Situation der Betroffenen. Der positiven Einflussnahme auf sozialrechtliche und medizinische Belange in der Gesetzgebung kommt eine immer stärker werdende und wachsende Bedeutung zu, um die Interessen von uns jungen Betroffenen bestmöglich $\mathrm{zu}$ vertreten und unsere Position nachhaltig zu verbessern. 


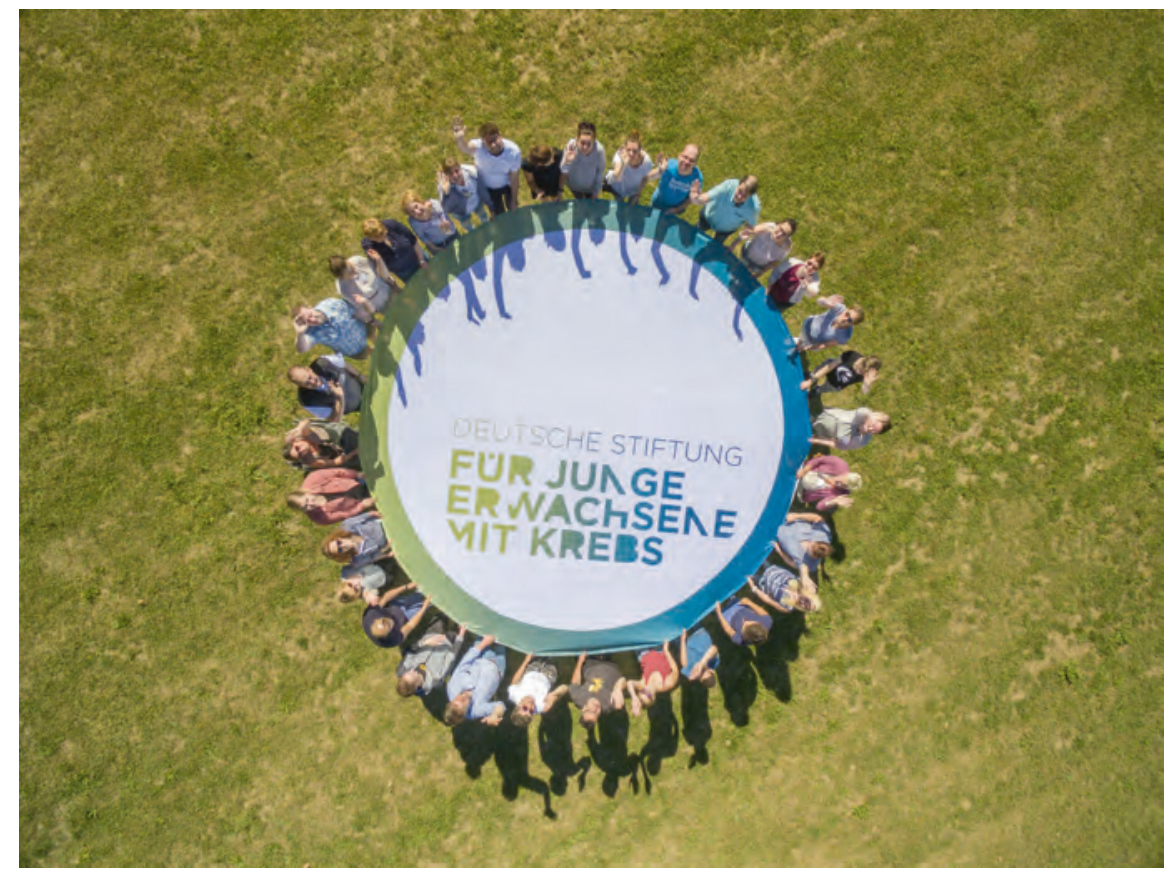

Abb. 1: Gemeinschaft erfahren - Austausch stärken: Gruppenbild des Jahrestreffens 2018 der bundesweiten TREFFPUNKTE der Deutschen Stiftung für junge Erwachsene mit Krebs in Lauterbach. Foto: Elmar Wiegand 